\title{
Volume Bragg Grating Locked Alexandrite Laser
}

\author{
Goronwy Tawy ${ }^{1, *}$, Ara Minassian ${ }^{2}$ and Michael J. Damzen ${ }^{1}$ \\ 1 Photonics Group, The Blackett Laboratory, Department of Physics, Imperial College London, Prince Consort \\ Road, London SW7 2AZ, UK; m.damzen@imperial.ac.uk \\ 2 Unilase Ltd., 60 Grays Inn Road, Unit LG04, London WC1X 8LU, UK; a.minassian@unilase.com \\ * Correspondence: goronwy.tawy12@imperial.ac.uk
}

check for updates

Citation: Tawy, G.; Minassian, A.; Damzen, M.J. Volume Bragg Grating Locked Alexandrite Laser. Optics 2022, 3, 53-59. https://doi.org/ $10.3390 /$ opt3010007

Academic Editors: Marco Lamperti and Riccardo Gotti

Received: 12 January 2022

Accepted: 1 February 2022

Published: 9 February 2022

Publisher's Note: MDPI stays neutral with regard to jurisdictional claims in published maps and institutional affiliations.

Copyright: () 2022 by the authors Licensee MDPI, Basel, Switzerland. This article is an open access article distributed under the terms and conditions of the Creative Commons Attribution (CC BY) license (https:// creativecommons.org/licenses/by/ $4.0 /)$.

\begin{abstract}
We report the first ever demonstration of a wavelength-locked Alexandrite laser using a volume Bragg grating (VBG) as a wavelength-selective mirror. Output power of $3.3 \mathrm{~W}$ with a diffraction limited beam quality of $\mathrm{M}^{2}=1.1$ was obtained at a lasing wavelength of $762.2 \mathrm{~nm}$ and a linewidth (FWHM) of $2.5 \mathrm{GHz}$.
\end{abstract}

Keywords: Alexandrite laser; diode-pumped solid-state laser; volume Bragg grating; tunable laser; narrow-linewidth laser

\section{Introduction}

Diode-pumped near-infrared vibronic lasers have gained growing interest over the last few years due to the development of high-power visible diodes. These include blue-diodepumped Ti:Sapphire [1] and red-diode-pumped $\mathrm{Cr}^{3+}$-doped materials such as Cr:LiSAF [2], Cr:LiCAF [3] and Alexandrite $\left(\mathrm{Cr}: \mathrm{BeAl}_{2} \mathrm{O}_{4}\right)$ [4]. The versatility of diode-pumped solidstate lasers in terms of low-cost, compactness and reliability has realised a number of applications in the 700-1000 $\mathrm{nm}$ region including spectroscopy [5], amplifier seeding [2] and remote sensing [6].

Red-diode-pumped Alexandrite has been shown to be a promising high-power laser source owing to its strong thermo-mechanical properties. For example, its thermal conductivity $\left(K_{c}=23 \mathrm{~W} \mathrm{~m}^{-1} \mathrm{~K}^{-1}\right.$ [7]) is over four times that of Cr:LiSAF and Cr:LiCAF [8]. Though there has been significant progress in blue-diode-pumped Ti:Sapphire lasers, the power levels have remained in general at around $<0.5 \mathrm{~W}$ with higher-power operation limited by parasitic issues [9]. These have been overcome but require complex multi-colourdiode arrangements [1] or green-diode-pumping which has achieved 3.8 $\mathrm{W}$ of output power but with a limited optical efficiency of 11.8\% [10]. Furthermore, Ti:Sapphire lasers are fundamentally limited by the larger quantum defect compared to red-diode-pumped Alexandrite lasers.

In our recent work, we achieved up to $7.4 \mathrm{~W}$ of output power and $>5 \mathrm{~W}$ at $755-780 \mathrm{~nm}$ with diffraction limited $\mathrm{TEM}_{00}$ mode quality [4]. Wavelength selection was obtained using an intracavity birefringent filter placed at Brewster's angle and rotated to the desired wavelength. For fixed wavelength operation, an alternative technique is to use a volume Bragg grating (VBG) as a wavelength-dependent highly reflective mirror [11]. This provides a well-defined wavelength selection as well as a narrow linewidth in a simple and compact system. In this work we present, to the best of our knowledge from the published literature (e.g., [12]), the first demonstration of a VBG-locked Alexandrite laser. Up to $3.3 \mathrm{~W}$ of output power at $762.2 \mathrm{~nm}$ with $2.5 \mathrm{GHz}$ linewidth (FWHM) is demonstrated from a compact and simple setup. This demonstrates the potential of diode-pumped Alexandrite lasers as high-power and narrow-linewidth near-infrared lasers for a wide range of applications. 


\section{Methods}

\subsection{Plane-Plane Diode-Pumped Alexandrite Laser}

Figure 1 shows the experimental setup for the diode-end-pumped Alexandrite laser. Pumping was provided by a continuous-wave $35 \mathrm{~W}$ multimode red-diode $\left(\lambda_{p}=640 \mathrm{~nm}\right)$ coupled into a $200 \mu \mathrm{m}$ diameter fibre $(\mathrm{NA}=0.22)$ and collimated using a $f_{c}=35 \mathrm{~mm}$ lens. The pump beam had a measured beam quality of around $\mathrm{M}^{2}=100$.

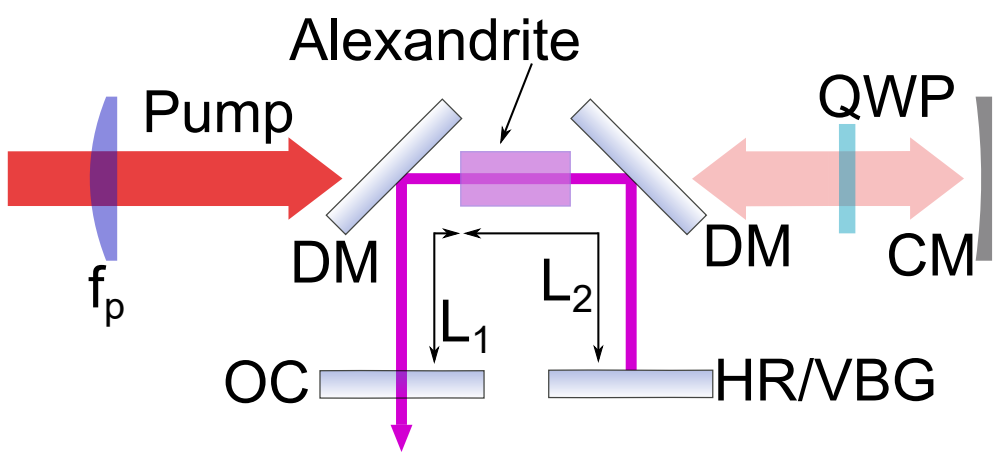

Figure 1. Four-mirror plane-plane cavity with double-pass end-pumping.

A four-mirror cavity was formed of: two dichroic mirrors (DM) that were highly transmissive (HT) at the pump wavelength and highly reflective at the laser wavelength $\left(\lambda_{l}=700-820 \mathrm{~nm}\right)$ at $45^{\circ}$; a mirror that was highly reflective at the laser wavelength at $0^{\circ}$ (HR); and an output coupler (OC) with reflectivity $R_{\mathrm{OC}}$. The HR mirror was later replaced by the volume Bragg grating (VBG). The cavity lengths $L_{1}=35 \mathrm{~mm}$ and $L_{2}=40 \mathrm{~mm}$ were set to provide a laser mode size of $\sim 200 \mu \mathrm{m}$ at the crystal for around $20 \mathrm{~W}$ of pumping based on the thermal lens modelling [13].

The gain medium was a $4 \times 4 \times 6 \mathrm{~mm}$ c-cut Alexandrite crystal with 0.2 at. $\%$ Cr-doping and anti-reflection coatings at both the pump and laser wavelengths. The crystal was mounted in a water-cooled copper manifold with the temperature at $25^{\circ} \mathrm{C}$. A $f_{p}=79 \mathrm{~mm}$ aspheric lens was used to focus the pump to a waist radius of $w_{p}=225 \mu \mathrm{m}$ (Rayleigh length of $z_{R}=2.5 \mathrm{~mm}$ ). The aspheric lens was positioned at $114 \mathrm{~mm}$ away from the fibre collimator to directly image the fibre output. At focus, the pump had a roughly top-hat intensity profile (Super-Gaussian of order $n=6$ ), which was chosen to minimise thermal aberrations.

The absorption coefficient of the crystal was measured to be $\alpha_{a} \approx 0.3 \mathrm{~cm}^{-1}$ and $\alpha_{b} \approx 6 \mathrm{~cm}^{-1}$ along the a-axis and b-axis of the crystal, respectively. The total single-pass absorption was measured to be around $75 \%$ with the residual pump light being almost entirely polarised along the a-axis. The residual pump light was retro-reflected using a concave mirror (CM) of curvature $R=100 \mathrm{~mm}$ and a quarter-waveplate was used to rotate the polarisation to the high-absorbing b-axis.

For wavelength selection and narrow-linewidth operation, the HR mirror was replaced by a volume Bragg grating (VBG). The VBG (Optigrate) had dimensions of $2 \times 2 \times 3.6 \mathrm{~mm}$ and a $>98 \%$ diffraction efficiency. The transmission of the VBG was measured using an in-house built narrow-linewidth Alexandrite laser (details of which can be found in [14]) with the results shown in Figure 2 and compared to the VBG data-sheet. The experiment confirmed the central wavelength to be $761.8 \mathrm{~nm}$ with a FWHM of around $0.3 \mathrm{~nm}$. The grating reflectivity was measured to be $>99 \%$ and it was decided to be used as an HR mirror. 


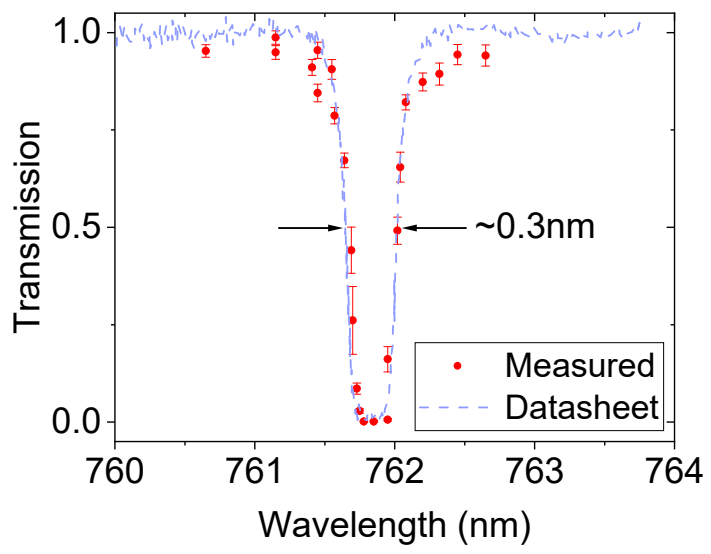

Figure 2. Measured VBG transmission spectrum.

\subsection{Convex-Plane Diode-Pumped Alexandrite Laser}

An alternative approach, in particular when considering power-scaling, is to use a convex mirror near the gain medium to counteract the strong thermal lens [4]. To assess the potential of this approach, a three-mirror cavity, as shown in Figure 3, was built. In this setup, the pump has been polarised prior to the $f_{p}=50 \mathrm{~mm}$ lens for single-end-pumping with around $17 \mathrm{~W}$ of incident power with a waist radius of $w_{p}=150 \mu \mathrm{m}$ (Rayleigh length of $z_{R}=1.0 \mathrm{~mm}$ ). The cavity was formed of a convex mirror of curvature $R=-300 \mathrm{~mm}$, which was HT at the pump wavelength and HR at the laser wavelength. The OC was placed at $45^{\circ}$ and the HR mirror (later replaced by the VBG) at normal incidence with $L_{1}=5 \mathrm{~mm}$ and $L_{2}=30 \mathrm{~mm}$ for optimal operation [4]. The aim of this setup was to assess the power-scaling performance with the VBG by considering the total output power. This setup could be easily altered for a single output, for example, by angling the convex mirror to form a four-mirror cavity.

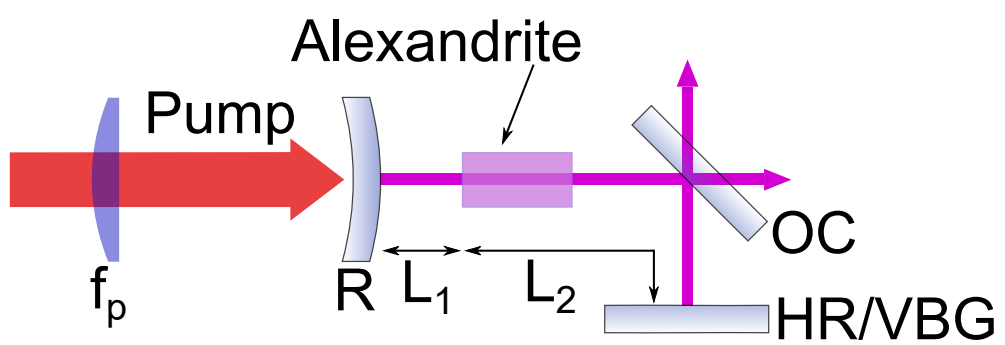

Figure 3. Three-mirror convex-plane cavity with single-end-pumping only.

\section{Results and Discussion}

\subsection{Plane-Plane Diode-Pumped Alexandrite Laser}

Figure $4 \mathrm{a}$ shows the laser power as a function of the absorbed pump power for the four-mirror plane-plane cavity without spectral control using the HR mirror. Two OCs were tested: $R_{\mathrm{OC}}=99 \%$ and $R_{\mathrm{OC}}=98 \%$. Optimal results were obtained with the former with a maximum laser power of $5.4 \mathrm{~W}$ at an absorbed pump power of $19.4 \mathrm{~W}$ (27.8\% optical efficiency) with a slope efficiency of $32.5 \%$ and threshold of $3 \mathrm{~W}$. The beam quality was measured to be $\mathrm{M}_{x}^{2}=1.5, \mathrm{M}_{y}^{2}=1.6$ (beam shown in inset of Figure $4 \mathrm{a}$ ). 

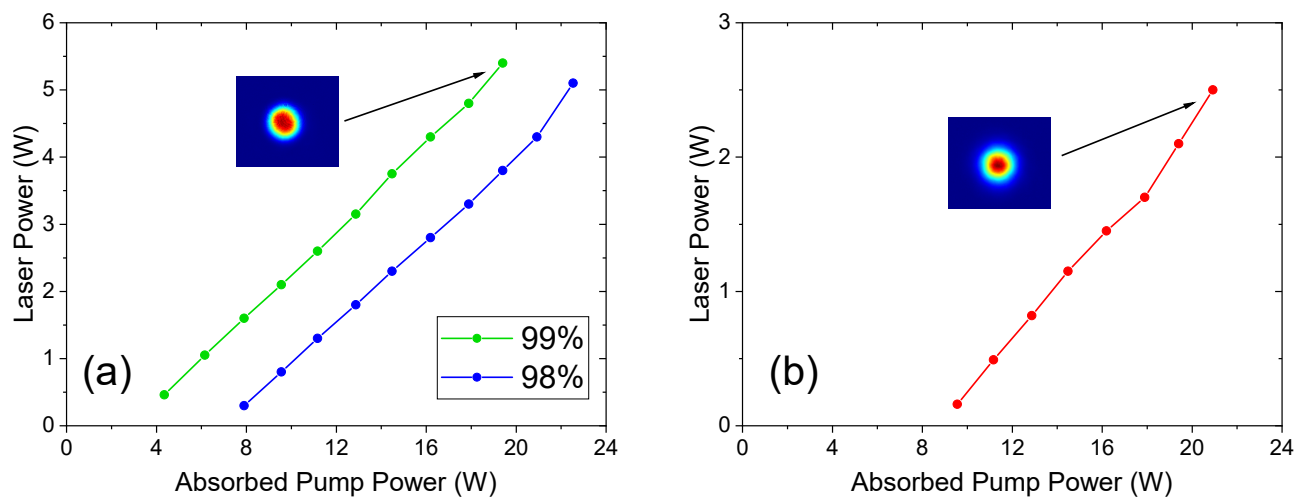

Figure 4. Laser power as a function of absorbed pump power for plane-plane cavity (a) with HR mirror (b) with VBG.

A further increase in laser power was obtained at higher pump power $(>19.4 \mathrm{~W})$ but with degradation in laser beam quality $\left(\mathrm{M}^{2}>2\right)$. This was attributed to the gradual mismatch between the pump and laser mode size at the gain medium. To investigate this, the beam width radius at the gain medium was measured from the DM leakage as a function of the absorbed pump power, and corrected to the TEM $\mathrm{T}_{00}$ beam width using the measured $\mathrm{M}^{2}$ values, with results shown in Figure $5 \mathrm{a}$. The reduction in beam width is consistent with a strengthening thermal lens (reduced focal length) as shown in Figure $5 \mathrm{~b}$. Better matching between the pump and laser modes at higher pump power is possible with higher-order modes, hence giving rise to the decrease in the laser beam quality. Improved beam quality can be obtained using a convex mirror and will be shown in the next section.
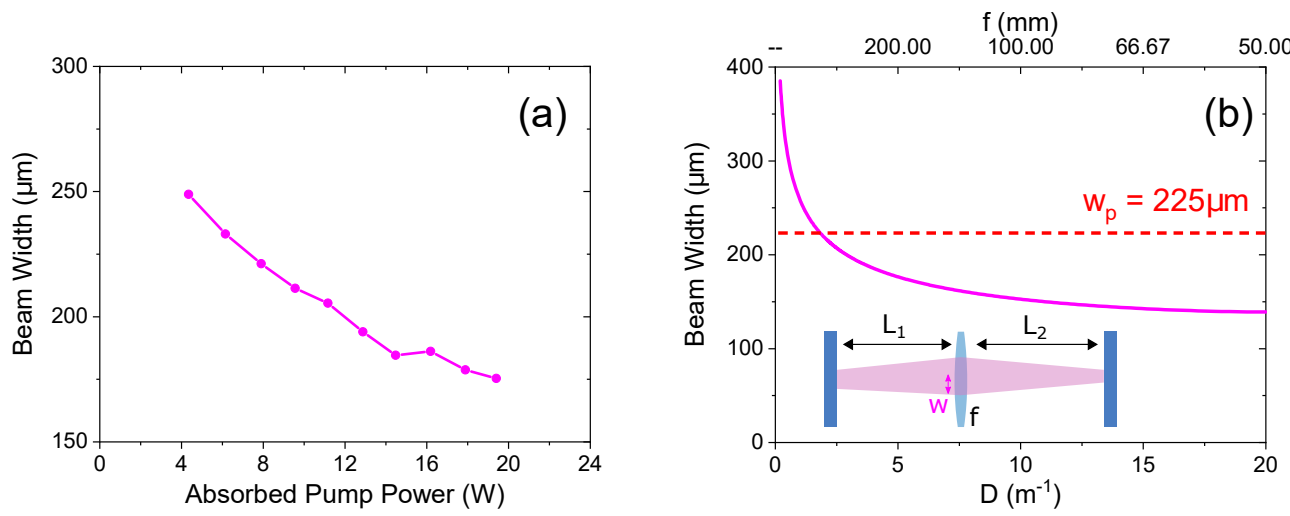

Figure 5. (a) Experimental $\mathrm{TEM}_{00}$ beam width radius (based on measured beam width and corrected using $\mathrm{M}^{2}$ value) as a function of the absorbed pump power for the plane-plane cavity. (b) Theoretical calculation of $\mathrm{TEM}_{00}$ beam width radius at the thermal lens as a function of its dioptric power $D=1 / f$ with $L_{1}=35 \mathrm{~mm}, L_{2}=40 \mathrm{~mm}$ and $\lambda=760 \mathrm{~nm}$.

Figure $4 \mathrm{~b}$ shows the laser power as a function of the absorbed pump power for the plane-plane cavity with the VBG and with $R_{\mathrm{OC}}=98 \%$ (which gave the optimal result). $2.5 \mathrm{~W}$ of laser power was obtained at $20.9 \mathrm{~W}$ of absorbed pump power (12\% optical efficiency) with a slope efficiency of $20.0 \%$ and threshold of $8.5 \mathrm{~W}$. Note that for the planeplane cavity without the VBG the threshold was around 7.1 W. The beam quality was excellent with $\mathrm{M}^{2}<1.1$ in both directions.

The laser spectrum was initially analysed using a grating spectrometer (resolution $\sim 0.1 \mathrm{~nm}$ ). Figure 6a shows the laser spectrum for the plane-plane cavity with and without the VBG. The free-running cavity (shown in light blue) has a broad and modulated spectrum. With the VBG (shown in red) the cavity is locked to a central wavelength of $762.1 \mathrm{~nm}$ - the small red-shift from the measured transmission in Figure 2 is possibly due to the temperature change, or a spectrometer calibration error. No significant change in 
wavelength with increased pump power was found and no damage was observed on the VBG throughout the experiment.

A $10 \mathrm{GHz}$ free-spectral range scanning Fabry-Perot interferometer (Finesse $>150$ ) was used to resolve the laser linewidth. Figure $6 \mathrm{~b}$ shows the results at the maximum laser power of $2.5 \mathrm{~W}$. The laser ran on three longitudinal modes with mode separation $\Delta v_{l} \approx 2 \mathrm{GHz}$ (which is consistent with the cavity length of around $L=L_{1}+L_{2}=75 \mathrm{~mm}$ ) and a FWHM linewidth $\Delta v \approx 2.5 \mathrm{GHz}$.
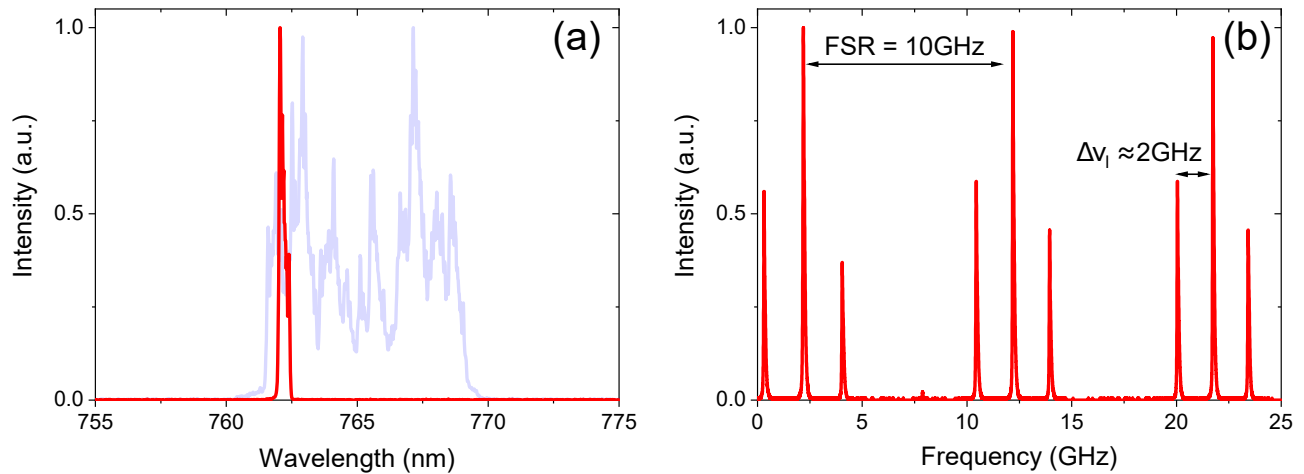

Figure 6. (a) Laser spectrum with (red) and without (blue) VBG. (b) Scanning Fabry-Perot interferometer demonstrating three longitudinal modes.

\subsection{Convex-Plane Diode-Pumped Alexandrite Laser}

Figure 7a shows the laser power (total output from OC) as a function of the absorbed pump power for the convex-plane cavity without spectral control. Three OCs were tested: $R_{\mathrm{OC}}=99 \%, R_{\mathrm{OC}}=98 \%$ and $R_{\mathrm{OC}}=97 \%$ with the highest power achieved with the latter. A maximum power of $5.2 \mathrm{~W}$ was achieved at an absorbed pump power of $15.7 \mathrm{~W}(33.1 \%$ optical efficiency) with a slope efficiency of $50.5 \%$ and threshold of $4.8 \mathrm{~W}$. The beam quality was measured to be $\mathrm{M}^{2}=1.1$ in both directions - demonstrating the improved performance of this cavity when power-scaling.
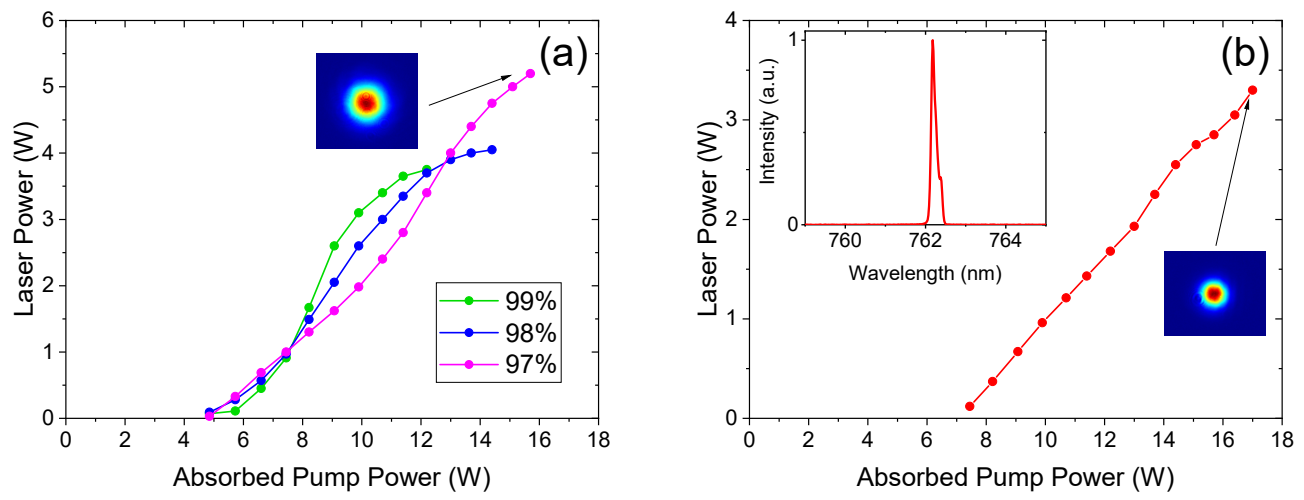

Figure 7. Laser power as a function of absorbed pump power for convex-plane cavity (a) with HR mirror (b) with VBG.

The HR mirror was replaced by the VBG for wavelength selection with $R_{\mathrm{OC}}=97 \%$. A maximum laser power of $3.3 \mathrm{~W}$ was achieved at an absorbed pump power of $17 \mathrm{~W}$ (optical efficiency of $19.4 \%$ ) with a slope efficiency of $33.4 \%$ and threshold of $7.2 \mathrm{~W}$, as shown in Figure $7 \mathrm{~b}$. These results show significant improvement in power and efficiency compared to the plane-plane cavity whilst maintaining an excellent beam quality of $\mathrm{M}^{2}=1.1$ in both directions. The laser operated at a wavelength of $762.2 \mathrm{~nm}$, as shown in the inset of Figure $7 \mathrm{~b}$. Using the scanning Fabry-Perot, it operated on two longitudinal modes with a linewidth of around $2.5 \mathrm{GHz}$. 


\section{Conclusions}

Diode-pumped Alexandrite lasers with the wavelength locked to a volume-Bragggrating (VBG) have been demonstrated for the very first time, to the best of our knowledge. This demonstrates the continued development in red-diode-pumped Alexandrite lasers as a highly promising low-cost and high-power near-infrared laser.

Using a four-mirror plane-plane cavity a maximum laser power of $5.4 \mathrm{~W}$ is obtained with $\mathrm{M}^{2} \leq 1.6$ with a broad and modulated spectrum. Replacing the broadband highly reflective mirror with the VBG locks and narrows the wavelength to $762.1 \mathrm{~nm}$ and $2.5 \mathrm{GHz}$, respectively, with an output power of $2.5 \mathrm{~W}$ and $\mathrm{M}^{2}<1.1$.

For power scaling with maintenance of high beam quality a convex-plane cavity was investigated with $5.2 \mathrm{~W}$ obtained with $\mathrm{M}^{2}=1.1$. With the VBG, $3.3 \mathrm{~W}$ was obtained at $762.2 \mathrm{~nm}$ with two longitudinal modes with a linewidth of around $2.5 \mathrm{GHz}$. The beam quality was also excellent with $\mathrm{M}^{2}=1.1$.

The system was limited by the available single-end-pumping power and further power scaling to $>5 \mathrm{~W}$ should be possible with retro-reflection or double-end-pumping [4]. VBG-locking could also be implemented into a unidirectional ring-laser design for singlelongitudinal-mode operation and $\sim \mathrm{MHz}$ linewidth operation. This could be easily implemented onto our previous ring-resonator designs [15-17].

Author Contributions: Conceptualization, M.J.D. and A.M.; methodology, M.J.D., A.M. and G.T.; software, G.T.; validation, G.T.; formal analysis, G.T.; investigation, G.T.; resources, M.J.D. and A.M.; data curation, G.T.; writing—original draft preparation, G.T.; writing—review and editing, G.T.; visualization, G.T.; supervision, M.J.D. and A.M.; project administration, M.J.D.; funding acquisition, M.J.D. All authors have read and agreed to the published version of the manuscript.

Funding: This research was funded by European Space Agency grant number 4000115840.

Institutional Review Board Statement: Not applicable.

Informed Consent Statement: Not applicable.

Data Availability Statement: The data that support the findings of this study are available from the corresponding author upon reasonable request.

Conflicts of Interest: The authors declare no conflict of interest.

\section{References}

1. Song, D.H.; Seo, H.S. Spectrally combined three-diode-pumped compact femtosecond Ti:sapphire laser exceeding $1 \mathrm{~W}$ modelocked power. Opt. Express 2021, 29, 32649-32657. [CrossRef] [PubMed]

2. Demirbas, U.; Thesinga, J.; Kellert, M.; Reuter, S.; Sumpf, B.; Pergament, M.; Kärtner, F.X. Mode-locked Cr:LiSAF laser far off the gain peak: Tunable sub-200-fs pulses near 1 m. Appl. Opt. 2021, 60, 9054-9061. [CrossRef] [PubMed]

3. Okuyucu, S.; Ozturk, Y.; Demirbas, U. Passively Q-switched Cr:LiCAF laser with a saturable Bragg reflector. Appl. Phys. B 2021, 127, 54. [CrossRef]

4. Tawy, G.; Minassian, A.; Damzen, M.J. High-power 7.4W TEM $\mathrm{T}_{00}$ and wavelength-tunable alexandrite laser with a novel cavity design and efficient fibre-coupled diode-pumping. OSA Contin. 2020, 3, 1638-1649. [CrossRef]

5. Sonnenschein, V.; Tomita, H.; Kotaro, K.; Koya, H.; Studer, D.; Terabayashi, R.; Weber, F.; Wendt, K.; Nishizawa, N.; Iguchi, T. A direct diode pumped Ti:sapphire laser with single-frequency operation for high resolution spectroscopy. Hyperfine Interact 2020, 241, 32. [CrossRef]

6. Munk, A.; Strotkamp, M.; Jungbluth, B.; Froh, J.; Mense, T.; Mauer, A.; Höffner, J. Rugged diode-pumped Alexandrite laser as an emitter in a compact mobile lidar system for atmospheric measurements. Appl. Opt. 2021, 60, 4668-4679. [CrossRef] [PubMed]

7. Walling, J.; Peterson, O.; Jenssen, H.; Morris, R.; O’Dell, E. Tunable alexandrite lasers. IEEE J. Quantum Electron. 1980, 16, 1302-1315. [CrossRef]

8. Demirbas, U. Power scaling potential of continuous-wave Cr:LiSAF and Cr:LiCAF lasers in thin-disk geometry. Appl. Opt. 2018, 57, 10207-10217. [CrossRef] [PubMed]

9. Moulton, P.F.; Cederberg, J.G.; Stevens, K.T.; Foundos, G.; Koselja, M.; Preclikova, J. Optimized InGaN-diode pumping of Ti:sapphire crystals. Opt. Mater. Express 2019, 9, 2131-2146. [CrossRef]

10. Miao, Z.W.; Yu, H.J.; Zou, S.Z.; He, C.J.; Zhao, P.F.; Lou, B.J.; Lin, X.C. Low-threshold-intensity 3.8-W continuous-wave Ti:Sapphire oscillator directly pumped with green diodes. Appl. Phys. B 2021, 127, 105. [CrossRef] 
11. Hemmer, M.; Joly, Y.; Glebov, L.; Bass, M.; Richardson, M. Volume Bragg Grating assisted broadband tunability and spectral narrowing of Ti:Sapphire oscillators. Opt. Express 2009, 17, 8212-8219. [CrossRef] [PubMed]

12. Sennaroglu, A.; Morova, Y. Divalent $\left(\mathrm{Cr}^{2+}\right)$, trivalent $\left(\mathrm{Cr}^{3+}\right)$, and tetravalent $\left(\mathrm{Cr}^{4+}\right)$ chromium ion-doped tunable solid-state lasers operating in the near and mid-infrared spectral regions. Appl. Phys. B 2022, 128, 9. [CrossRef]

13. Tawy, G.; Wang, J.; Damzen, M.J. Pump-induced lensing effects in diode pumped Alexandrite lasers. Opt. Express 2019, 27, 35865-35883. [CrossRef] [PubMed]

14. Tawy, G.; Damzen, M.J. Tunable, dual wavelength and self-Q-switched Alexandrite laser using crystal birefringence control. Opt. Express 2019, 27, 17507-17520. [CrossRef] [PubMed]

15. Sheng, X.; Tawy, G.; Sathian, J.; Minassian, A.; Damzen, M.J. Unidirectional single-frequency operation of a continuous-wave Alexandrite ring laser with wavelength tunability. Opt. Express 2018, 26, 31129-31136. [CrossRef] [PubMed]

16. Sathian, J.; Tawy, G.; Sheng, X.; Minassian, A.; Damzen, M.J. Nonastigmatic alexandrite ring laser design with wavelength-tunable single-longitudinal-mode operation. J. Opt. Soc. Am. B 2020, 37, 2185-2192. [CrossRef]

17. Tawy, G.; Minassian, A.; Damzen, M.J. 8.5 W Linear and 3.6 W Ring TEM 00 Diode-Pumped Alexandrite Lasers. In Proceedings of the 2021 Conference on Lasers and Electro-Optics Europe European Quantum Electronics Conference (CLEO/Europe-EQEC), Munich, Germany, 21-25 June 2021. 